\title{
Menschenrechte als Übersetzungsproblem
}

\author{
von Doris Bachmann-Medick
}

\begin{abstract}
Investigations of current and historical human rights discourses gain new perspectives when viewed as a problem of translation: by examining non-European transformations/displacements/revisions of the universal principles of the UNDeclaration (1948), critical implementations of these principles in local practices and - almost more importantly - re-translations of these local transformations into new declarations of human rights principles. The article discusses the complex conditions under which the universal claim of a western human rights discourse could be challenged by considering translational activities which attempt to identify new, but common reference points for a transcultural human rights discourse.
\end{abstract}

\section{Ein „Translational Turn“?}

Die anhaltende Debatte über Menschenrechte hat mittlerweile eine komplexe und konfliktreiche interkulturelle Auseinandersetzung ausgelöst. Umstritten sind vor allem die universelle Geltung und transkulturelle Begründbarkeit der Menschenrechte, aber auch ihre praktischen Umsetzungen, Transformationen und offensichtlichen Verletzungen. Diese Auseinandersetzung könnte im Licht der Übersetzungsdiskussion in den Kultur- und Sozialwissenschaften einen neuen konzeptuellen Angelpunkt gewinnen: Menschenrechte entfalten ihr Potential als ein Übersetzungsproblem.

Quer durch die Disziplinen der Kulturwissenschaften richtet sich die Aufmerksamkeit gegenwärtig verstärkt auf die Kategorie der Übersetzung. Das traditionelle Übersetzungsverständnis im Sinn von (philologischer und linguistischer) Text- und Sprachübersetzung wird dabei entschieden erweitert. Zunehmend ist von kultureller Übersetzung die Rede. Doch damit ist allzu oft nur eine metaphorische Begriffsverwendung verbunden, die sich in letzter Zeit geradezu inflationär ausbreitet. Wie aber ließe sich „Übersetzung“ von einer derart ausufernden Metapher zu einer eigenständigen Analysekategorie entfalten? Eine Möglichkeit wäre, Übersetzung als soziale und (inter)kulturelle Praxis auszuarbeiten und sie damit zu konkretisieren: als Vermittlung oder Verhandlung, als Sinnverschiebung und Transformation durch Übertragungen in neue Kontexte hinein. Übersetzung wäre damit direkter im Feld von Handlungen und Interaktionen anzusiedeln. Mit einem derart praxisorientierten Übersetzungsverständnis scheint die kultur- und sozialwissenschaftliche Forschung gegenwärtig auf die unübersehbaren Differenzen, Verwerfungen und Asymmetrien in einer globalisierten Welt zu reagieren, die ihrerseits auf Übersetzungsprozesse angewiesen sind: auf Übersetzungen zwischen sozialen und ethnischen Gruppen, zwischen Religionen, zwischen 
kulturellen Systemen, zwischen Wissenschaftstraditionen und Wissenskulturen. Herausgefordert durch die gesellschaftlichen, kulturellen und politischen Handlungswelten selbst, wird Übersetzung zugleich ein wichtiger Fokus für deren wissenschaftliche Analyse.

Wenn die Kulturwissenschaften ihre Aufmerksamkeit gegenwärtig verstärkt auf „Übersetzung“ richten, dann meinen sie also beides: eine Praxiskategorie, ausdrücklich aber auch eine epistemologisch geschärfte Analysekategorie. Damit legen sie ein wichtiges Scharnier frei, das die Forschungssphäre an die gesellschaftspolitische Sphäre rückbindet. Zwar sollten in den Kulturwissenschaften immer die Alarmglocken läuten, wenn Kategorien der gesellschaftlichen Handlungswelten, wie beispielsweise Identität, Nation oder Grenze, gleichzeitig auch als analytische Kategorien übernommen werden. Doch im Fall der Übersetzungskategorie besteht offensichtlich eine geringere Gefahr, dass die Forschung durch solche Kategorienübernahme wiederum unreflektierte gesellschaftliche Wahrnehmungen und Klassifizierungen reifiziert. Denn bereits auf der gesellschaftlichen Ebene erweist sich Übersetzung als eine komplexe Kulturtechnik, mit der auch in schwierigen Kontakt- und Handlungssituationen durchaus selbstreflexiv mit Differenzerfahrungen, Code- und Ebenenwechseln umgegangen werden kann. Sie bezieht sich auf Aushandlungs- und Vermittlungsschritte, Adressierungen und Transformationsvorgänge. Auf der Ebene der Analyse wiederum entspricht einer solchen Kulturtechnik die Forschungseinstellung einer „methodischen Interkulturalität“.

In diesem Sinn meint Übersetzung als Analysekategorie: komplexe gesellschaftliche Problemfelder, kulturelle Kontakte und Konflikte, ja überhaupt die Übergänge zwischen unterschiedlichen Kontexten handlungsanalytisch aufzugliedern. Gerade die vielschichtige Dynamik der Akteure ist hierbei genauer in den Blick zu rücken - ihre diskursiven und medialen Einbindungen, ihre jeweiligen Bezugssysteme und Referenzrahmen, ihr Umgehen mit Differenzen, kurz: ihre Übersetzungsakte, wie sie an den einzelnen Schritten und Schichten ihrer Interaktionen besonders greifbar werden. Eine derart translatorische Durchleuchtung richtet sich gleichermaßen auf vereinheitlichende Allgemeinbegriffe wie etwa Identität, Nation, Kultur, Gesellschaft, Religion. Sie ist im Spiel, wenn Jürgen Habermas von Religionsgemeinschaften in postsäkularen Gesellschaften fordert, dass sie ihre religiöse Sprache in eine öffentlich zugängliche säkulare Sprache hinein „übersetzen“ müssen, wenn ihre Anlie-

1 Martin Fuchs, Interkulturelle Hermeneutik statt Kulturvergleich. Zur sozialen Reflexivität der Deutungsperspektiven, in: Ilja Srubar u. a. (Hg.), Kulturen vergleichen. Sozialund kulturwissenschaftliche Grundlagen und Kontroversen, Wiesbaden 2005, S. $112-150$, hier S. 115. 
gen Gehör finden sollen. ${ }^{2}$ Sie ist ebenfalls am Werk, wenn der Soziologe Joachim Renn eine ganze Soziologie auf „Übersetzungsverhältnisse“ begründet, indem er „Übersetzung“ zu einer gesellschaftstheoretischen Schlüsselkategorie ausarbeitet - womit er neue Zugänge eröffnet zur Praxis von Beziehungen und Grenzüberschreitungen zwischen sozialen Gruppen in Prozessen gesellschaftlicher Integration. ${ }^{3}$ In der Geschichtswissenschaft hält die Übersetzungskategorie - wie der Forschungsbericht in der Einleitung zu diesem Themenheft zeigt - schon jetzt auf zahlreichen Feldern Einzug und ermöglicht dort genauere Untersuchungen von Übergängen, Vermittlungen, Missverständnissen und Brechungen: besonders im Feld von Migration und kulturellen Grenzgängern, von Mission und Konversion, von komplexen Kulturtransfers sowie kolonialen und postkolonialen Transformationen. ${ }^{4}$ Gerade für eine transnationale Geschichtsschreibung wird es unverzichtbar, nach kulturellen Übersetzbarkeiten, Unübersetzbarkeiten oder epistemologischen Ungleichheiten bereits im Feld der Untersuchungsbegriffe selbst zu fragen. ${ }^{5}$ Auch die Disziplin der Translation Studies reicht neuerdings tiefer als bisher in gesellschaftspolitische Übersetzungssphären hinein. Titel wie "Translating Terror", 6 „Violence and Translation", 7 ,Translation and Conflict" sind nur einige der zahlreichen Beispiele für neue translatorische Forschungs-

2 Jürgen Habermas, Religion in der Öffentlichkeit. Kognitive Voraussetzungen für den „öffentlichen Vernunftgebrauch" religiöser und säkularer Bürger, in: ders., Zwischen Naturalismus und Religion. Philosophische Aufsätze, Frankfurt 2005, S. 119-154.

3 Joachim Renn, Übersetzungsverhältnisse. Perspektiven einer pragmatistischen Gesellschaftstheorie, Weilerswist 2006.

4 Vgl. u.a. Peter Burke u. R. Po-chia Hsia (Hg.), Cultural Translation in Early Modern Europe, Cambridge 2009; Vicente L. Rafael, The Promise of the Foreign. Nationalism and the Technics of Translation in the Spanish Philippines, Durham 2005; Douglas R. Howland, Translating the West. Language and Political Reason in Nineteenth-Century Japan, Honolulu 2002; vgl. das Themenheft von WerkstattGeschichte 48. 2008 zum Über-Setzen; siehe auch die Beiträge dieses Heftes.

5 Margrit Pernau, Transkulturelle Geschichte und das Problem der universalen Begriffe. Muslimische Bürger im Delhi des 19. Jahrhunderts, in: Birgit Schäbler (Hg.), Area Studies und die Welt. Weltregionen und neue Globalgeschichte, Wien 2007, S. 117 - 149; vgl. dies., Bürger mit Turban. Muslime in Delhi im 19. Jahrhundert, Göttingen 2008; ferner Monica Juneja u. Margrit Pernau, Lost in Translation. Transcending Boundaries in Comparative History, in: Heinz-Gerhard Haupt u. Jürgen Kocka (Hg.), Comparative and Transnational History. Central European Approaches and New Perspectives, New York 2009, S. $105-129$.

6 Susan Bassnett, Translating Terror, in: Third World Quarterly 26. 2005, S. 393-403.

7 Veena Das, Violence and Translation, in: Anthropological Quarterly 75. 2002, S. $105-112$.

8 Mona Baker, Translation and Conflict. A Narrative Account, London 2006. 
felder. ${ }^{9} \mathrm{Ob}$ man jedoch wirklich so weit gehen sollte, von einem „translational turn ${ }^{\text {"10 }}$ zu sprechen, hängt davon ab, wieweit sich Übersetzung in empirischen Fallstudien über eine bloße Metapher hinaus als eine methodisch einsetzbare Analysekategorie bewährt, mit der komplexe Zusammenhänge auf ihre einzelnen Übersetzungsschritte hin ausgelotet werden können. ${ }^{11}$

Ein Aufbruch in diese Richtung ist der Versuch, die Übersetzungsspielräume von Akteuren gerade auch in problematischen Situationen des Kulturenkontakts auszuloten: bei Hindernissen und Störungen im Umgang mit kulturellen Differenzen, bei interreligiösen Konflikten, aber auch in kulturinternen Integrationsvorhaben sogenannter multikultureller Gesellschaften. Missverständnisse oder gar Brüche sind hier schon deshalb ernster zu nehmen als bisher, weil Verwerfungen zugleich auch konstruktive Ausgangspunkte für interkulturelle Verständigungsversuche sein können. Dies wird allerdings erst erkennbar, wenn man bereit ist, eine harmonisierende Vorstellung vom Übersetzen als Brückenbauen zwischen Kulturen aufzugeben.

Dieses immer noch weit verbreitete Brücken-Modell könnte nun einer konfliktbewussteren translatorischen Analysehaltung weichen. So steht Übersetzen immer deutlicher für eine Praxis des Aushandelns von Differenzen, wie sie gerade in kulturellen Zwischenräumen aufbrechen. Doch auch diese Einstellung neigt dazu, sich wiederum in einer jargonglatten Formel zu verfestigen. Zwar wertet sie ausdrücklich die „shifts“ oder gar Brechungen zwischen unterschiedlichen Lebensweisen, Artikulationssphären, Diskursformen, Kulturen oder Wissenschaftssystemen auf, um diese als Kontaktzonen analytisch fruchtbar zu machen. Doch verliert sie sich dabei allzu leicht in einer Pauschalvorstellung von Kulturübersetzung, wie sie noch in Homi Bhabhas griffig-spielerischer Formulierung durchscheint: „Culture [...] is both transnational and translational“. ${ }^{12}$ Auch mit dieser „Formel“ lässt sich erst dann analytisch arbeiten, wenn sie ihrerseits translatorisch aufgebrochen wird. Denn die (kulturellen) Zwischenräume, auf die sie anspielt, erschüttern ja nicht schon aus sich heraus das Regime bipolarer Grenzziehungen wie etwa wir - die Anderen oder das Eigene - das Fremde. Nötig wäre hier vielmehr, zu den praktischen Gestaltungsräumen von Beziehungen vorzudringen: zu Akteuren sowie zu konkreten Übersetzungsprozessen in Kontakt- und Übergangsszenarien. Dafür wären die von den Kulturwissenschaften so

9 Aufschluss über die Entwicklung geben auch zwei neu gegründete Zeitschriften: Translation Studies (London, seit 2008) sowie Translation. A Transdisciplinary Journal (Manchester, seit 2011).

10 Vgl. Doris Bachmann-Medick (Hg.), The Translational Turn (= Themenheft Translation Studies 2. 2009); ferner dies., Translational Turn, in: dies., Cultural Turns. Neuorientierungen in den Kulturwissenschaften, Reinbek $2010^{4}$, S. $238-283$.

$11 \mathrm{Zu}$ den methodischen Voraussetzungen und Kriterien für einen „turn“ vgl. BachmannMedick, Cultural Turns, S. 26 f.

12 Homi K. Bhabha, The Location of Culture, London 1994, S. 172. 
gefeierten „Zwischenräume“ einmal wirklich als konkrete Handlungs-, ja Übersetzungsräume zu untersuchen. Statt also transnationale Beziehungen und Verflechtungen pauschal als „hybride“ Überlappungszusammenhänge zu behaupten, würden diese dann als ein Ergebnis von Übersetzungsvorgängen auch tatsächlich nachweisbar. In der Frage der Menschenrechte scheint es ebenfalls verkürzt, deren universellen Geltungsanspruch allein schon aus hybriden Verflechtungsbeziehungen abzuleiten. Im Gegenteil, eine allzu glatte Annahme kultureller „Hybridisierungen“ ist auch hier translatorisch in Frage zu stellen.

Übersetzung als Analysekategorie wird auch der Menschenrechtsdebatten auf eine Ebene vorstoßen müssen, auf der zuallererst die unterschiedlichen Ausgangsbedingungen und sperrigen Tiefenstrukturen der wissenschaftlichen, kulturellen oder politischen Kommunikation und Interaktion kritisch beleuchtet werden können. Hierzu wäre die Übersetzungsperspektive so weit auszudehnen, dass sie auch die Analyseinstrumentarien der eigenen Forschungstradition einer kritischen Selbstreflexion aussetzt und deren Übersetzbarkeit prüft. Immerhin legt sie ein grundlegendes Forschungsdilemma frei, von dem nicht zuletzt die Frage der Übersetzbarkeit der Menschenrechte angesichts unterschiedlicher Rechtskulturen betroffen ist: „,man übersetzt das Fremde direkt in die eigenen Begriffe und Rechtsbegriffe, und was da nicht hinein passt, wird abgeschnitten " ${ }^{13}$ So erscheint es unabdingbar, bereits die verwendeten Analysebegriffe und Konzepte selbst ausdrücklich zu lokalisieren und zu historisieren. Denn entgegen ihrem oft universalen Geltungsanspruch können sie doch auf ihre westlichen und damit durchaus beschränkten Entstehungskontexte und „origin points“ zurückverfolgt werden. Dadurch wird eine „cross-categorical translation “14 praktiziert, die viel weiter reicht als eine „cross-cultural translation“. Denn mit ihr könnten die Theorien, Konzepte und Analysebegriffe des eigenen Wissenschaftssystems wenigstens ansatzweise mit den Schlüsselbegriffen anderer, auch nicht-westlicher Wissenschaftskulturen „verhandelbar" gemacht werden. ${ }^{15}$

Die translatorische Analysehaltung wäre also bis auf das eigene Forschungsvokabular selbst auszudehnen. Dies hieße - auch für eine Problematisierung der Menschenrechte im Horizont von Übersetzung - nach Gelenkstellen für "cross-categorical translations" zu suchen und eben nicht davon auszugehen, dass Menschenrechte gleichsam wie ein "travelling concept" weltweit zur

13 Franz von Benda-Beckmann, Übersetzung, Vergleich, Transformation. Das lästige Recht der Anderen, Antrittsvorlesung 7.7.2005 an der Universität Halle-Wittenberg, http:// www.eth.mpg.de/cms/en/people/d/fbenda/pdf/antrittsvorlesungHalle.pdf.

14 Dipesh Chakrabarty, Provincializing Europe. Postcolonial Thought and Historical Difference, Princeton 2000, S. 83-86.

15 Zur „Politik des Übersetzens“ in diesem Zusammenhang vgl. Dipesh Chakrabarty, Europa als Provinz. Perspektiven postkolonialer Geschichtsschreibung, Frankfurt 2010, S. 14. 
Geltung gebracht werden könnten. Anregend hierfür ist ein Vorschlag von rechtsanthropologischer Seite: die „Entwicklung einer analytischen Vergleichssprache, mit deren Hilfe man Gemeinsamkeiten und Unterschiede zwischen unterschiedlichen empirischen Rechtsformen (folk systems) beschreiben, vergleichen und analysieren kann". ${ }^{16}$ Da dieser Vorschlag die jeweiligen Rechtskulturen historisiert und sie aus den historischen Konstellationen des Kolonialismus herleitet, wird er auch für die Geschichtswissenschaft relevant. Noch weiter führen würde er allerdings, wenn man ihn nicht nur auf die Untersuchung der Übersetzungsbedingungen in einem rechtspluralistischen Umfeld bezieht, sondern ihn komplexer entfaltet für die übergreifende Frage nach Menschenrechten als Übersetzungsproblem. Jedenfalls legt die weltweite Auseinandersetzung mit der umstrittenen Autorität einer westlich formulierten Menschenrechtserklärung nahe, verdeckte Schichten des Übersetzungsprozesses genauer zu beleuchten: Vorübersetzungen, die auf der Ebene der unterschiedlichen Referenzbezüge angesiedelt sind, Selbstübersetzungen, die mit Lokalisierungen einhergehen sowie Wechselübersetzungen zwischen globalen Rechtsansprüchen und lokalen Rechtskontexten.

\section{Menschenrechte in translatorischer Sicht}

Auch Menschenrechte reisen nicht etwa von sich aus schon als ein „travelling concept", sondern werden immer erst durch Akteure in lokale Verhältnisse und Vorstellungswelten hinein „übersetzt“. Strittig ist jedoch nicht nur diese praktische Umsetzung der Menschenrechte, die ja häufig gerade durch deren Verletzung gefordert ist. Strittig ist vor allem die transkulturelle Begründbarkeit der Menschenrechte. Darüber ist seit einigen Jahrzehnten eine globale Auseinandersetzung ausgebrochen, die freilich nicht nur auf der diskursivargumentativen Ebene eines „Austauschs von Gründen“"17 stattfindet. Die globalen Menschenrechtsdebatten sind gerade auch durch ein komplexes Hin und Her in den Transfers und Anwendungen der Menschenrechtsidee geprägt, durch global-lokale Zirkulationen und wechselseitige Übertragungsprozesse. Sie könnten daher unter translatorischem Vorzeichen neu gesehen und

16 Benda-Beckmann, Übersetzung, Vergleich, Transformation, S. $7 \mathrm{f}$.

17 Vgl. Regina Kreide, Globale Politik und Menschenrechte. Macht und Ohnmacht eines politischen Instruments, Frankfurt 2008, S. 205. Mit Blick auf die „Mikroebene der Argumentation“ (S. 206) schlägt Regina Kreide hier das auf Argumenten beruhende „deliberative Modell“ (S. 201) als Muster der Konsensbildung über Menschenrechte vor. Es könnte vielleicht durch ein Übersetzungsmodell ergänzt werden, das - über die Begründungsebene hinaus - die Mikroebene von transnationalen Anwendungen, Wechselübertragungen und Zirkulationen zwischen globalen und lokalen Arenen der Menschenrechtsdebatte noch stärker mit einbezieht. 
bewertet werden: als ein spannungsreiches Übersetzungsszenarium. Hiermit betritt man allerdings Neuland.

Noch heute ist der durchgehende Bezugspunkt der Menschenrechtsdebatte die 1948 von den United Nations verabschiedete Universal Declaration of Human Rights (UDHR). Diese Erklärung ist in ihren 379 Sprachen zum meistübersetzten Dokument der Welt geworden: „the document most translated indeed the most ,universal " - in the world“, ausgezeichnet mit dem „Guinness World Record“, wie es die Human Rights Website der UN stolz verkündet. ${ }^{18}$ Hier, im Feld der Sprachübersetzung, meldet sich also unverkennbar die Autorität eines Text-„,Originals“, das allein schon auf der Ebene der Textübertragung massive sprachliche Übersetzungsprobleme aufgeworfen hat. ${ }^{19}$ Und doch liegen die entscheidenden Übersetzungsherausforderungen gerade nicht auf der Textebene, sondern im kulturellen Feld - ausgehend von der Frage: Gibt es eine transkulturelle Grundlegung der Menschenrechte? Verkörpert die emphatisch-initiale Menschenrechtsdeklaration auch in Bezug auf diese Frage das „Original“, auf das die folgenden Erklärungen, Überarbeitungen und Übersetzungen aus den unterschiedlichsten Weltregionen bis heute rückbezogen bleiben?

Die gegenwärtige weltweite Menschenrechtsdebatte besteht - so provokant es klingen mag - aus Übersetzungen ohne Original. Dies ist schon deshalb der Fall, weil die UN-Deklaration von 1948 selbst zwar mit der Autorität eines „Originals“ aufgetreten ist, doch ihrerseits bereits Übersetzungen von Grundpositionen der aufklärerischen Menschenrechtserklärungen des 18. Jahrhunderts enthält. Sie übernahm nicht einfach die klassischen Grundrechte dieser frühen Menschenrechtserklärungen, sondern übersetzte sie in zeitgemäßere und konkretere Rechtsfelder hinein, etwa in das Recht auf Familie oder das Recht auf Arbeit. Mit einem translatorischen Rückblick lässt sich allerdings wiederum erkennen: Auch schon die französische Erklärung der Menschenund Bürgerrechte, die Déclaration des droits de l'homme et du citoyen von 1789, war kein abgeschlossener Gründungstext, sondern geradezu ein performativer Entwicklungsanstoß - nicht zuletzt kraft ihrer kommunikationsstimulierenden "religiösen Kodierung“. ${ }^{20}$ Außerdem beruhte auch diese Gründungserklärung bereits auf zahlreichen französischen Übersetzungen der vorhergehenden amerikanischen Declaration of Independence von $1776 .{ }^{21}$

18 http://www.ohchr.org/EN/UDHR/Pages/WorldRecord.aspx.

19 Hierzu vgl. Marianne Garre, Human Rights in Translation. Legal Concepts in Different Languages, Kopenhagen 1999.

20 Vgl. Martin Schaffner, Menschenrechte und Demokratie. Über die Globalisierung von Sinn, in: Brigitte Hilmer u. a. (Hg.), Anfang und Grenzen des Sinns. Für Emil Angehrn, Weilerswist 2006, S. 264-272.

21 Vgl. Lynn Hunt, Inventing Human Rights. A History, New York 2007, S. 127; vgl. auch Marcel Gauchet, Menschenrechte, in: François Furet u. Mona Ozouf, Kritisches 
Auch griff sie die politischen Rechteforderungen der Cahiers de Doléances auf, der Beschwerdehefte aus den Provinzen, die sie dann in einem spiralförmigen Prozess des Hin- und Herübersetzens zwischen Verfassungs-, Natur- und Menschenrechten wiederum auf eine radikalere Stufe trieb. ${ }^{22}$ Schließlich entfaltete sich die Déclaration zwischen 1789 und 1795 - eingelassen in heftige zeitgenössische Debatten über den Einschluss oder Ausschluss bestimmter sozialer Gruppen wie Protestanten, Juden, Frauen oder Sklaven $-{ }^{23}$ sodann ihrerseits zu einem ausdifferenzierten Übersetzungsergebnis des revolutionären Prozesses selbst. Sie führte zu Neufassungen und zu neuen Verfassungen: zur Verfassung von 1791 und zu neuen sozialen Rechten in der jakobinischen Verfassung von 1793. In einem grundlegenden Vortrag, der jüngst in der deutsch-französischen Online-Zeitschrift Trivium wiederabgedruckt wurde, ${ }^{24}$ hat Étienne Balibar gezeigt, wie dabei das klassische Naturrecht als Referenzpunkt abgelöst und in Sozialrechte übersetzt wurde. Eine derartige Übersetzung entpuppte sich in der zeitgenössischen Auseinandersetzung als deutliche Politisierung. Denn bereits um 1789 - so Balibar wurde eine „unbegrenzte Sphäre der Politisierung von Rechtsansprüchen“25 eröffnet, aus der dann jeder Arbeiter, jede Frau, bis hin zu Sklaven und Kolonisierten rechtliche Ansprüche ableiten konnten. Ich würde ergänzen: Es wurde ein bis heute unabgeschlossener Prozess der Menschenrechtstranslation in Gang gesetzt. Dieser sorgt auch heute noch dafür, dass menschenrechtliche Ansprüche fortwährend politisch angemeldet werden können.

Zwar ist in einer gewissen historischen Kurzsichtigkeit nicht etwa diese klassische französische Menschenrechtserklärung zum Hauptbezugspunkt der heutigen Menschenrechtsdiskussion gemacht geworden, sondern die chronologisch jüngere UN-Erklärung der Nachkriegszeit von $1948 .{ }^{26}$ Ein für sich stehendes „Original“ ist aber auch diese nicht. Eher verkörpert sie einen allgemeinen Referenzpunkt, ein modellhaftes „document of intent“, ${ }^{27}$ einen Kanon von universalen Prinzipien, Normen und Absichtserklärungen. Eben deshalb war und ist man hier auf kulturell konkretisierende Übersetzungsleistungen angewiesen. Menschenrechte, so meine These, bilden sich über-

Wörterbuch der Französischen Revolution, Bd. 2, Frankfurt 1996, S. 1180 -1197, hier S. $1182-1185$.

22 Zur Rolle der Cahiers vgl. Jürgen Sandweg, Rationales Naturrecht als revolutionäre Praxis. Untersuchungen zur „Erklärung der Menschen- und Bürgerrechte“ von 1789, Berlin 1972, S. 121 - 156. Zum Spiralmodell des Übersetzens siehe unten.

23 Hunt, Inventing Human Rights, S. 146 - 175.

24 Étienne Balibar, Die Proposition Égaliberté („Gleichfreiheit“), in: Trivium 3. 2009, http://trivium.revues.org/3337.

25 Ebd., S. 13.

26 Zur Entstehungsgeschichte, Bedeutung und historischen Nachwirkung der französischen Menschenrechtserklärung vgl. Schaffner, Menschenrechte und Demokratie.

27 Garre, Human Rights in Translation, S. 15. 
haupt erst durch komplexe kulturelle Übersetzungsprozesse heraus. Menschenrechtsübersetzung - heißt dies etwa nur praktische Umsetzung, Aneignung, Transformation oder Verletzung? Weit wirkungsvoller erweisen sich die diversen Versuche, den aktuellen globalen Menschenrechtsdiskurs auf fortdauernde interkulturelle Normenbildungsprozesse hin offen $\mathrm{zu}$ halten und damit dessen Befangenheit in der europäisch-humanistischen Menschenrechtstradition zu überwinden. Dies verlangt freilich, ausdrücklich wechselseitige Übersetzungsprozesse in den Blick zu nehmen. Und solche kommen ans Licht, wenn man die unterschiedlichen Ebenen des Menschenrechtsdiskurses in ihren Verflechtungen freilegt: die Ebenen der normativen Deklaration, der pragmatisch-politischen Lokalisierung, aber auch die Spannungsräume und Spielräume interkultureller Aushandlung.

\section{Normative Deklaration: Divergierende Menschenrechtserklärungen}

Dass also die Universal Declaration of Human Rights von 1948 zum Schlüsseltext für die gegenwärtige Menschenrechtsdebatte werden konnte, liegt nicht nur an ihrem universalen Geltungsanspruch, sondern auch an der kontroversen Übersetzungsgeschichte, die sie ausgelöst hat: kritische Übersetzungsprozesse zwischen westlichen und nicht-westlichen Menschenrechtspositionen, von Ergänzungsvorschlägen bis hin zu Gegenentwürfen. Doch bereits der Entstehungsprozess der UDHR selbst war ein höchst komplexer und widersprüchlicher Übersetzungsvorgang. Nach einem etwa zweijährigen Aushandlungsprozess waren die Vertreter von westlichen Gesellschaften, aber auch von Indien, China, Iran, Libanon, Ägypten, Syrien, Sowjetunion, Philippinen, Uruguay, Chile, Kuba, Brasilien - unter Vorsitz von Eleanor Roosevelt - letzten Endes zur Konsensbildung aufgerufen: ${ }^{28}$ Die gemeinsame Stoßrichtung damals war - unter dem Eindruck des Zweiten Weltkriegs, von Faschismus und Holocaust -, staatliche Menschenrechtsverletzungen $\mathrm{zu}$ verhindern und das Individuum ausdrücklich vor Staatswillkür zu schützen. In dieser Stoßrichtung schlug sich allerdings eine deutlich westliche Prägung

28 Übersetzungsprobleme traten bereits in der Entwurfsphase der UDHR häufig auf. Exemplarisch ist hierfür eine Passage aus dem Protokoll der 22. Sitzung am 5. Mai 1948: „Mr. Hendrick (United States of America) and Mr. Santa Cruz (Chile) stated that in the legal systems of their countries, the word ,person' could have various interpretations, and this might cause difficulties in translation." Meeting Records des Drafting Committee, http://daccess-dds-ny.un.org/doc/UNDOC/DER/NL4/803/76/ PDF/NL480376.pdf?OpenElement. Ein weiteres von unzähligen Beispielen zu Debatten über „translation“ und „wording“ ist die Auseinandersetzung über den Gebrauch der Wörter „cruel and inhuman“ in Artikel 7: „Mr. Wilson (United Kingdom) said that he would abstain from voting on Article 7 as it stood because the words ,cruel ${ }^{\text {c }}$ and ,inhuman' were too subjective. What might be called,cruel or inhuman' in one country might not be considered so in another", http://daccess-dds-ny.un.org/doc/UNDOC/ DER/NL4/803/85/PDF/NL480385.pdf?OpenElement. 
nieder, die auch die vorherrschenden Freiheits- und Gleichheitsrechte betraf, die Individualrechte und liberalen Vorstellungen sowie die Überzeugung vom grundrechtsadäquaten Prinzip der Demokratie. Insgesamt zeichnete sich das Menschenrechtskonzept der UDHR von 1948 dann auch durch ein ausgesprochen säkulares und liberales Vorzeichen aus. Es waren gerade diese unbefragten Voreinstellungen, welche schon in der Vorbereitungsphase dieser UN-Erklärung die American Anthropological Association dazu veranlassten, aus kulturanthropologischer Sicht massive Kritik zu äußern: ${ }^{29}$ Es wurde bemängelt, die UDHR sei trotz ihres universalen Anspruchs unverkennbar mit den Prinzipien des westlichen Liberalismus verklammert. Sie sei auf ein autonomes Individuum fixiert, ohne dessen Einbindung in soziale Gruppen zu berücksichtigen. Sie ignoriere die Existenz kultureller Differenzen und könne deshalb keineswegs bruchlos für andere Kulturen und Gesellschaften geltend gemacht werden.

Diese Kritik aus den späten 1940er Jahren argumentierte allerdings noch strikt kulturrelativistisch - entlang eines zu jener Zeit stark essentialistischen, auf kulturelle Identität pochenden Kulturverständnisses. In der Zwischenzeit hat sich die Lage grundlegend verändert. Nicht nur haben sich die wechselseitigen globalen Abhängigkeiten verschärft; auch die kulturtheoretischen Diskurse haben darauf mit einem zunehmend hybridisierten Verständnis von Kulturen geantwortet: Die Einsicht in kulturelle Überlappungen und kulturinterne Widersprüchlichkeiten lässt kulturrelativistische Rückzugspositionen heutzutage jedenfalls kaum mehr zu. In der Tat hat vor allem in den letzten zwanzig Jahren die Vielstimmigkeit im Menschenrechtsdiskurs zugenommen, ohne dass aber die nach wie vor universalistische Menschenrechtsformulierung kulturrelativistisch unterhöhlt worden wäre. Eher wurden die Einwände aus den unterschiedlichen Weltregionen derart in die Debatte eingebracht, dass diese für die Möglichkeit einer internationalen Konsensbildung gerade durch die Artikulation von Differenzen weiterhin offen gehalten wird.

So wurde eine translatorische Dynamik in Gang gesetzt, die sich nun an wichtigen Stationen der interkulturellen Auseinandersetzungen über Menschenrechte genauer aufzeigen lässt. Diese Stationen sind bahnbrechend für den Versuch, Menschenrechte künftig nicht primär westlich, sondern eher multilokal und dadurch auf neue Weise global zu verankern. Erstaunlich ist, dass dabei schon auf der Ebene der Deklaration selbst, in der Übernahme oder auch Transformation von normativen Menschenrechtsstandards, unentwegt brisante kulturelle Übersetzungsbeziehungen am Werk sind. Es ist ein translatorisches Spannungsfeld, das sich hier zwischen den voneinander abweichenden europäischen, asiatischen, afrikanischen, islamischen Systemen auftut: Eine dieser zentralen Stationen bildet die afrikanische Menschen-

29 Vgl. The Executive Board, American Anthropological Association, Statement on Human Rights, in: American Anthropologist, New Series 49. 1947, S. $539-543$. 
rechtserklärung, die African (Banjul) Charter on Human and Peoples' Rights von $1981 .{ }^{30}$ Sie ist ein klares Ergebnis des Dekolonisierungsprozesses und der postkolonialen Selbstartikulation. Als erstes warf sie ein kritisches Licht auf die UDHR selbst, die sie explizit als ein Übersetzungsprodukt vorangehender, noch versteckter Kolonial- und Imperialismusbestrebungen entlarvte. Hier hakte die Banjul-Charta mit ihrer nicht-europäischen Perspektive ein, um die „universalen“ Menschenrechte zu ergänzen und umzudeuten. Dies geschah in erster Linie dadurch, dass kollektive Rechte gegen die Vorherrschaft von Individualrechten ins Feld geführt wurden. Ausdrücklich - und das ist neu wurden in Artikel 29.5 auch Pflichten eingefordert und von regionalen Standpunkten aus formuliert: insbesondere die Pflicht „to preserve and strengthen the national independence“. Eine so angelegte Übersetzung des Menschenrechtsvokabulars ist nun alles andere als ein Rückbezug auf ein Original. Eher setzt sie sich über dessen Entstehungseinflüsse und Referenzbezüge - wie westlichen Kolonialismus und Imperialismus sowie implizite christlich-jüdische Werte - kritisch hinweg. Andere, postkoloniale Referenzbezüge werden dem entgegengesetzt.

Menschenrechtstranslation steht hier deutlich unter dem Vorzeichen postkolonialer Brüche. Es liegt nahe, von diskontinuierlicher Übersetzung zu sprechen, von „translation-as-displacement“," wie Dipesh Chakrabarty die Verwerfungen in Übersetzungsprozessen aufgrund postkolonialer Machtasymmetrien bezeichnet hat. Schließlich wird in der Präambel auf nationalkulturelle „independence“ ebenso rekurriert wie auf „peoples” rights“ (statt nur individueller Rechte), auf Ausrottung von jeglichem Kolonialismus sowie auf ein konstitutives „right to development“. Während die UN-Declaration aufgrund ihrer europäisch-humanistischen Referenz bürgerliche und politische Rechte isolierte, wird in der afrikanischen Präambel ausdrücklich hervorgehoben "that civil and political rights cannot be dissociated from economic, social and cultural rights". Und so sind es auch diese letzteren Rechtstypen, die in der afrikanischen Erklärung überwiegen. Die 1988 für ihre praktische Umsetzung geschaffene African Commission sorgte darüber hinaus für weitere Konkretisierungen - etwa $\mathrm{zu}$,the free operation of trade unions and the right to strike“. So heißt es in einem Kommentar mit ausdrücklichem Übersetzungsbezug:

The African Commission could begin by initiating a process of imaginative translation of the bare rights in the Charter into appropriate frameworks for implementation. The Commission can begin to approach this issue by borrowing approaches to the issue developed by

30 Unter:http://www.africa-union.org/official_documents/Treaties_\%20Conventions_\%20 Protocols/Banjul\%20Charter.pdf.

31 Dipesh Chakrabarty, Place and Displaced Categories; Or, How We Translate Ourselves into Global History, in: Doris Bachmann-Medick u. a. (Hg.), The Trans/National Study of Culture, Berlin [2013]. 
both its regional counterparts - the Inter American Commission and Court on Human Rights and the European Union system - and translating those approaches in the context of existent conditions on the continent. ${ }^{32}$

Was die Banjul-Charta auszeichnet, ist ihre entschiedene Referenz auf den afrikanischen „liberation struggle against foreign domination“ (Artikel 20.3). Damit ist bereits auf der Ebene der Deklaration, also noch vor jeglicher praktischen Umsetzung, eine politisierende Übersetzung am Werk - auch wenn mit der Orientierung an "the values of African civilization“ (Präambel) ein essentialisierender Zug ins Spiel kommt. Bereits eine solche Zuschreibung von kulturellen Wesensmerkmalen ist ein Anzeichen dafür, dass hier die universalistische Weichenstellung des neueren Menschenrechtsdiskurses verlassen wird.

Ähnliche Lokalisierungen wenn nicht gar Essentialisierungen auf der Ebene der Übersetzung von Normensystemen wurden auch von asiatischer Seite aus eingebracht. So mischte sich in den 1990er Jahren die Debatte über „asiatische Werte“ (im Unterschied zu „europäischen Werten“) in den immer komplexer werdenden transkulturellen Übersetzungsprozess ein. Diese vor allem auch innerasiatische Debatte führte wiederum andere Menschenrechtsprinzipien ins Feld: Gemeinschaftsrechte wurden gegen individuelle Rechte angeführt, order und discipline nach dem konfuzianischen System stärker betont als liberty und freedom - wenngleich, wie der indische Ökonom und Entwicklungsphilosoph Amartya Sen hervorhebt, solche Abweichungen immer auch schon die westlichen wie asiatischen Traditionen selbst durchzogen haben. ${ }^{33}$ Prägendes Vorzeichen ist jedenfalls ein paternalistisches Staatsverständnis, das allerdings auch die Funktion haben kann, die eigene, autoritäre, staatlich repressive Regierungsform zu legitimieren und zu kaschieren, was sich bis heute - etwa in Chinas Menschenrechtsgebahren - bemerkbar macht. Überhaupt ist auffällig, wie massiv von asiatischer Seite aus am Prinzip staatlicher Souveränität („national sovereignty“) festgehalten wird, vor allem am Prinzip der Nichteinmischung in die Angelegenheiten der jeweiligen Staaten („non-interference in the internal affairs of States"). Herausgehoben wird aber auch die Notwendigkeit, politische Rechte stets Hand in Hand mit der - für Asien ja höchst dynamischen - ökonomischen Entwicklung zu formulieren. So jedenfalls die Bangkok Declaration, ${ }^{34}$ die als asiatischer Beitrag zur World Conference on Human Rights von 1993 in Wien zu verstehen ist - nicht hingegen als offizielle Menschenrechtserklärung, wie sie von

32 http://wwwl.umn.edu/humanrts/africa/Oloka-Onyango.html.

33 Vgl. Amartya Sen, Universal Truths. Human Rights and the Westernizing Illusion, in: Harvard International Review 20. 1998, S. 40-43, http://www.mtholyoke.edu/acad/ intrel/asian\%20values/sen.htm.

$34 \mathrm{http}: / /$ law.hku.hk/lawgovtsociety/Bangkok\%20Declaration.htm. 
asiatischer Seite aus bis heute nicht ausformuliert worden ist. Auch hier wurde und wird der Universalitätsanspruch der UN-Erklärung unterlaufen.

Noch eine dritte, dezidierte Menschenrechtstranslation lässt sich als ein wichtiges Glied in diese transkulturelle Übersetzungskette einreihen: Etwa zur gleichen Zeit, 1990, folgte nämlich die Erklärung der fünfzig Mitgliedsstaaten der Islamischen Konferenz, die sogenannte Cairo Declaration on Human Rights in Islam. ${ }^{35}$ Diese „islamische“ Menschenrechtserklärung betont in einem Akt religiöser Übersetzung: die „Islamic Schari'ah is the only source of reference for the explanation or clarification of any of the articles of this Declaration“ (Art. 25). Von Übersetzung kann hier kaum mehr die Rede sein, eher vom Versuch einer Revision der UN-Erklärung. Revidiert wird hier allein schon das säkulare Konzept der Menschenrechte zugunsten einer explizit religiösen Grundlegung. Und relativiert wird damit auch der Anspruch auf universelle Geltung der Menschenrechte. Durch Berufung auf die Schari'ah bleibt vielmehr die Geltung auf islamische Gesellschaften beschränkt, wird Egalität in Bezug auf Nicht-Muslime und Frauen in Frage gestellt, der Gleichberechtigung der Religionen wird widersprochen und Religions- wie Meinungsfreiheit explizit beschnitten. ${ }^{36}$

Somit ist fraglich, ob diese islamische Revision der UN-Erklärung überhaupt als ein Übersetzungsakt gelten kann. Denn es wird ja nicht etwa eine kulturspezifische Lokalisierung universaler Grundsätze vorgenommen. Vielmehr wird eine kulturrelativistisch anmutende Position universalistisch verkleidet und so wiederum dem westlichen Universalisierungsanspruch entgegengehalten. Entscheidend aber ist, dass dabei ein völlig anderer Referenzpunkt zur Geltung gebracht wird. Gilt für die UN-Erklärung trotz ihres christlich-jüdischen Kontexts grundsätzlich ein säkular-liberaler Referenzpunkt: säkulare Rechte, so setzt die Islamische Erklärung mit der Schari'ah als religiös-islamischem Rechtssystem einen gänzlich anderen Referenzpunkt ins Werk: religiöse Verpflichtungen. Hier geht es also weniger darum zu „übersetzen“ als vielmehr zwei divergierende Referenzsysteme "gegeneinander zu setzen“. ${ }^{37}$ Schon von daher wäre es verkürzt, diesen

35 http://wwwl.umn.edu/humanrts/instree/cairodeclaration.html.

36 Vgl. - auch kritisch - Heiner Bielefeldt, Menschenrechte in der islamischen Diskussion, http://www.kompetenz-interkulturell.de/userfiles/Grundsatzartikel/Menschenrechte\%20Islam.pdf; Christine Schirrmacher, Islamische Menschenrechtserklärungen und ihre Kritiker - Einwände von Muslimen und Nichtmuslimen gegen die Allgültigkeit der Scharia, 2008, http://www.igfm.de/index.php?id=1035.

37 Die Aushebelung des westlichen Referenzsystems ist auch der gemeinsame Nenner einer innerarabischen Debatte um Menschenrechte, vgl. Burhan Ghalyoun, Human Rights in Contemporary Arabic Thought, in: Salma K. Jayyusi (Hg.), Human Rights in Arab Thought. A Reader, London 2009, S. 343 -373, hier S. 362: „The common denominator among all these, however, is the wish to take the concept out of its western framework and modify it in line with the values of Arab Islamic culture.“ 
wichtigsten von mehreren noch folgenden islamischen Gegenentwürfen zur UN-Erklärung als „Herausforderung für den christlich-islamischen Dialog“38 zu bezeichnen. Eine Übersetzungsperspektive greift gerade hinter eine solche Engführung auf eine interreligiöse Kommunikationsebene zurück, um von dort darüber hinaus zu führen. Ein derartiger „Dialog“ kann nämlich erst dann funktionieren, wenn man bereits im Vorfeld ansetzt und die Ebene der „pretranslation“ ausdrücklich in den Übersetzungsprozess mit einbezieht: die Ebene unterschiedlicher Referenzsysteme. Mit einem solchen Fokus auf „pretranslations" würde die Aufmerksamkeit nicht zuletzt auch auf innerkulturelle Debatten gelenkt - etwa auf innerislamische, wie sie bereits in den arabischen Gesellschaften selbst über die Frage der Menschenrechte und ihre Universalität ausbrechen.

Schon deshalb erschöpft sich eine Übersetzungsperspektive der Menschenrechte nicht in dem Befund, dass diese jeweils in christliche, asiatische oder islamische Werte umgedeutet beziehungsweise in verschiedenen Weltregionen auf je besondere, abweichende Weise angeeignet werden. Der Blickwinkel der Translation legt vielmehr komplexere Vermittlungen und Ebenenwechsel frei, die für die Menschenrechtsdebatte und ihre Analyse genutzt werden können. Zum einen gibt der Übersetzungsfokus zu erkennen, wie unverzichtbar es ist, auf die Ebene der „pre-translation“ vorzustoßen, eben auf unterschiedliche Referenzsysteme. Und hier liegen meines Erachtens die entscheidenden Gelenkstellen oder „shifts“ zwischen Akzeptanzen, Widerständigkeiten oder Wechselübersetzungen in den kulturellen Wanderungen und Abwandlungen von Menschenrechtspositionen. Zum andern sind nach Meinung des portugiesischen Sozialtheoretikers Boaventura de Sousa Santos gerade die Unvollständigkeiten (und damit die Ergänzungsbedürftigkeit) der jeweiligen kulturellen Positionen und Konzeptionen als produktive Anstöße in der Menschenrechtsdebatte anzuerkennen:

The recognition of reciprocal incompleteness and weakness is a conditio-sine-qua-non of a cross-cultural dialogue. The work of translation builds both on local identification of incompleteness and weakness and on its translocal intelligibility. ${ }^{39}$

Die verflochtene Kette der Menschenrechtsübersetzungen erscheint schon von daher in neuem Licht: als „partial translations“ von „universal declarations“ wobei die universalen Positionen gerade nicht in einem (westlichen) „Original“ (der UN-Erklärung) verkapselt sind, sondern sich als noch zu profilierende Zielprojektion einer globalisierten Menschenrechtsdebatte abzeichnen.

38 Heiner Bielefeldt, Moderne Menschenrechte als Aufgabe für Christen und Muslime, http://www.flw.ugent.be/cie/bielefeldt3.htm.

39 Boaventura de Sousa Santos, The Future of the World Social Forum. The Work of Translation, in: Development 48. 2005, S. 15 - 22, hier S. 18, http://www.ces.uc.pt/myces/ UserFiles/livros/55_The_Future_of_the_WSF_Development_2005.pdf. 


\section{2. "Partial Translations“}

In welchem Sinn ist hier eigentlich von „translations “ und noch dazu von „partial“ translations die Rede? Die durchaus harte „Arbeit der Übersetzung“ zu verfolgen, scheint jedenfalls den global vermehrten Konfliktlagen angemessener zu sein als ein harmonistisches Vorverständnis von „interkulturellem Dialog“ zugrundezulegen, das Differenzen leicht überspielt. Denn die Frage der Menschenrechte einer differenzbewussten Übersetzungsperspektive auszusetzen, heißt gerade: sich einlassen auf unvermeidliche Kommunikations- und Vermittlungsspannungen. Ein solcher Ansatz wird dann produktiv, wenn auch er nicht von vornherein auf vollständige oder gar gelungene Übertragungen fixiert bleibt.

Richtungweisend wäre hier eine Einsicht des Ethnologen James Clifford, der ähnlich wie Boaventura de Sousa Santos darauf verweist, dass wissenschaftliche Kulturanalysen, aber auch das kulturelle Wissen selbst, unweigerlich selektiv, unvollständig, standortgebunden und von Machtbezügen durchsetzt sind. Solche „partial truths“ anzuerkennen, hieße, gerade damit den Prozessen der Produktion von Kultur (noch vor ihrer Interpretation) auf die Spur zu kommen. ${ }^{40}$ Sind nicht analog dazu auch im Feld der Formulierung und Umsetzung der Menschenrechte in erster Linie partial translations zu erwarten, die aber auch hier ein erhebliches strategisches Potential entfalten? Auch hier könnten kulturelle Differenzen - so ließe sich behaupten - besonders durch partial translations für Handlungsanstöße offen gehalten werden. Jedenfalls könnten sie zum Einfallstor werden für ein gezielt „untreues“ Aneignungsverhalten gegenüber dem „Original“ („faithless appropriation"), ${ }^{41}$ das ausdrücklich Umdeutungen produktiv werden lässt. Denn statt auf ein „Original“ der Menschenrechtserklärung fixiert zu bleiben, eröffnen sie vielmehr neue Handlungsoptionen und Aneignungsmöglichkeiten, die sich auch den Äquivalenzzwängen einer bisher westlich monopolisierten Übersetzungshoheit verweigern könnten. Erst dadurch ließen sich „vielstimmige“ Perspektiven für den Menschenrechtsdiskurs freisetzen: in Aussicht auf eine keineswegs glatte transnationale Verhandlungskonstellation, aus der heraus überhaupt erst gemeinsame (allgemeine, normative) „Referenzpunkte“ angepeilt werden könnten. Ein solcher Übersetzungsprozess, in den sich außereuropäische Weltregionen als unverzichtbare Akteure einbringen, bleibt auf jeden Fall unabgeschlossen.

Auch wenn Menschenrechte lokal implementiert werden, lässt dies also noch längst nicht darauf schließen, dass damit der Übersetzungsprozess der Menschenrechte abgeschlossen wäre. Der nur partielle Modus von Menschenrechtsübertragungen wirkt auch hier gewissermaßen wie ein Stachel und Ansporn. Denn er verweist die jeweiligen Gesellschaften darauf, dass jenseits aller lokalen

40 James Clifford, Introduction. Partial Truths, in: ders. u. George E. Marcus (Hg.), Writing Culture. The Poetics and Politics of Ethnography, Berkeley 1986, S. 1-26, hier S. 1 u. S. 7.

41 Anna Lowenhaupt Tsing, Transitions as Translations, in: Joan W. Scott u.a. (Hg.), Transitions, Environments, Translations. Feminisms in International Politics, New York 1997, S. 253 -272, hier S. 253. 
Aneignungs- und Implementierungsprozesse ein anhaltender, umfassenderer Referenzrahmen genutzt werden kann, der wie eine Messlatte für die konkrete Menschenrechtspolitik vor Ort wirkt.

Auch die „partiellen“ lokalen Menschenrechtsforderungen selbst bleiben schon deshalb unabgeschlossen und damit anhaltender wirksam, weil sie an spezifische Rechtsfelder geknüpft sind, die sie dann wiederum in neue allgemeine Menschenrechtsformulierungen „einspeisen“ können - man denke etwa an eingeforderte Rechte indigener ethnischer Gruppen, an Partizipationsansprüche ärmster Bevölkerungsschichten in kommunalen Entscheidungsprozessen, an das Recht auf Entwicklung, an Rechte von Frauen und deren Schutz vor Diskriminierung und Gewalt, an Kinderrechte, Rechte von Minoritäten oder Verdammung von Folter. Wie eine solche „Einspeisung“ von partikularen Menschenrechtsforderungen in den allgemeinen Erklärungen zu Buche schlägt, zeigt sich besonders an der schon erwähnten Wiener World Conference on Human Rights von 1993. ${ }^{42}$ Deren Sinn war es ja geradezu, unter Beteiligung von 171 Nationen und vor allem unter dem Druck von 400 NGOs die noch allzu formelhaft-allgemeinen Menschenrechtserklärungen im Gefolge der UN-Deklaration zu bestätigen, sie dann aber doch auf solche spezifischen Rechtsfelder hin noch weiter auszudifferenzieren.

Zwar sprach die von der Konferenz verabschiedete Wiener Erklärung (Vienna Declaration and Programme of Action) das schon existierende internationale Geflecht von Kooperationen und Beziehungen an. Und dieses sei - wie es im achten Abschnitt dieser Erklärung heißt - ausdrücklich auf ein „promoting of democracy“ zu verpflichten, gerade auch für „least developed countries“. Von solchen kulturimperialistischen Anklängen einmal abgesehen, wurde aber insgesamt nicht etwa eine westliche Einbahnstraße der Menschenrechtsübersetzung eingeschlagen, keine lineare Schiene, die nur von der Normenebene auf die lokale Umsetzungsebene führt. Hier wurden vielmehr Wege gebahnt für eine folgenreiche Wechselübersetzung durch außereuropäischen „Gegenverkehr“: ${ }^{33}$ „Regional arrangements play a fundamental role in promoting and protecting human rights“, heißt es im Abschnitt 37 der Wiener Erklärung. Vor allem also sind es die lokalen und regionalen Handlungsfelder, die dementsprechend als komplexe „Übersetzungsräume“ ernst zu nehmen sind, zumal sie den Aushandlungsprozess von Menschenrechten in besonderer Weise vorantreiben könnten. Zwar erscheinen sie auf den ersten Blick begrenzt: als bloße Schauplätze einer örtlichen Aneignung der Menschenrechtsdiskurse. Und doch vermitteln sie weit darüber hinaus entscheidende Impulse für Rückübersetzungen in die allgemeine, normative Sphäre der Menschenrechtserklärungen hinein.

42 http://www.unhchr.ch/huridocda/huridoca.nsf/\%28Symbol\%29/A.CONF.157.23.En.

43 Peter Sloterdijk, Im Weltinnenraum des Kapitals. Für eine philosophische Theorie der Globalisierung, Frankfurt 2006, S. 222. 


\section{Lokalisierung und globale Rückübersetzung}

Menschenrechte verlangen bekanntlich mehr als nur eine weltweite, universale Anerkennung. Unverzichtbar wird es, sie in lokale Rechtsvorstellungen sowie in landessprachliche Denk- und Handlungswelten hinein zu übertragen, damit sie für lokale Akteure Sinn erhalten: „In order for human rights ideas to be effective, however, they need to be translated into local terms and situated within local contexts of power and meaning ${ }^{\text {(44 }}$ - so die Rechtsanthropologin Sally Merry in ihrem Buch, das Menschenrechte und Translation bereits im Titel verknüpft: „Human Rights and Gender Violence. Translating International Law into Local Justice“ ". ${ }^{45}$ Eine solche „vernacularization on the ground“ bedarf allerdings entsprechender Übertragungsvorgänge und „channels and technologies of transmission " ${ }^{46}$ um allgemeine, erklärte Menschenrechte an die Lebensumstände der Menschen anzuschließen und sie damit wirkungsvoller in soziale Aktivitäten überführen zu können.

Was aber heißt dies konkret? Fallstudien wie die hier angedeutete aus Uttar Pradesh zeigen, wie in dieser indischen Region arme, kastenlose Frauen selbst ihr „empowering ${ }^{\text {“47 }}$ in die Hand nehmen, gerade wenn es um „translating human rights concerning violence against women ${ }^{\text {“48 }}$ geht. Dabei wirken Frauenkollektive als Instanzen von Menschenrechtsübersetzung, indem sie normative Forderungen für die eigene Situation umdeuten und in lokale Handlungswelten übertragen. Übersetzung von Menschenrechten meint hier also zunächst Lokalisierung - eine Grundbedingung für die effektive Umsetzung globaler Vorstellungen in Praktiken am Ort. Die Aufmerksamkeit darauf zu richten, wie abstrakte Rechtsnormen in kulturspezifische Normen, Traditionen und lokale Diskurse übertragen werden, heißt: Mikroprozesse der Vermittlung zu erkennen - also Verständigungsprozesse, aber auch Missverständnisse, Widerstände und Machtbehauptungen beim Versuch, Menschenrechte am Ort durchzusetzen. Nur so lässt sich Zugang finden zu den konkreten Vermittlern, die auf sehr unterschiedlichen Ebenen agieren: von NGOs bis hin zu lokalen Initiativen und grassroot-Aktivisten wie Frauengruppen. Solche Menschenrechtsaktivisten sind hier auf je eigene Weise als Mittler und Übersetzerinnen zwischen den universellen Ansprüchen sowie den lokalen Bedrohungen und Entrechtungen tätig: „Their translation work

44 Sally Engle Merry, Human Rights and Gender Violence. Translating International Law into Local Justice, Chicago 2006, S. 1.

45 Ebd.

46 Vgl. Peggy Levitt u. Sally Merry, Vernacularization on the Ground. Local Uses of Global Women's Rights in Peru, China, India and the United States, in: Global Networks. A Journal of Transnational Affairs 9. 2009, S. 441 -461, hier S. 441.

47 N. Rajaram u. Vaishali Zararia, Translating Women's Human Rights in a Globalizing World. The Spiral Process in Reducing Gender Injustice in Baroda, India, in: Global Networks. A Journal of Transnational Affairs 9. 2009, S. 462 -484, hier S. 469.

48 Merry, Human Rights, S. 138. 
involves bringing the content of rights discourse to new areas, such as domestic violence and property“. 49

Bei genauerem Hinsehen zeigt sich nun, dass selbst solche soziale Übersetzungsarbeit noch deutlich angewiesen ist auf sprachliche Übersetzung. Immerhin nutzt sie je nach Kontext ganz unterschiedliche rhetorisch-diskursive Übersetzungsstrategien und kulturspezifische Narrative. So wird beispielsweise ein explizites Menschenrechtsvokabular eingesetzt, wenn man sich an ein internationales Publikum, Programm oder auch an Sponsoren wendet. Ein anderes, moralisches Vokabular wird wiederum verwendet, um auf der lokalen Ebene Anschluss zu finden an kulturelle Traditionen und Praktiken. ${ }^{50}$ Übersetzung erweist sich hier also nicht zuletzt als ein ausdrückliches Ansprechen unterschiedlicher Adressaten und damit als ein sprachliches Changieren zwischen den diversen Arenen des Menschenrechtsdiskurses. Geht es zum einen darum, das Menschenrechtsidiom gezielt für lokale Redeweisen verfügbar $\mathrm{zu}$ machen, wird damit immer auch schon die Möglichkeit geschaffen, von den lokalen Ungleichheiten aus (neue) Elemente und Forderungen in den allgemeinen Menschenrechtskatalog zurückzuprojizieren.

Eine solche mehrpolige Übersetzungspraxis ist ein entscheidender Ansatzpunkt, um in einer entstehenden Weltgesellschaft von einseitigen Übersetzungsrichtungen abzurücken und ausdrücklich wechselseitige Übersetzungen $\mathrm{zu}$ ermöglichen. Wechselseitig deshalb, weil einerseits die Nutzung des globalen Menschenrechtsdiskurses auch für marginalisierte Gesellschaften gleichsam das Eingangstor zur „modernen“, westlichen Welt und zur globalen Zivilgesellschaft bildet. Andererseits wird dadurch wiederum die globale Sphäre des Menschenrechtsdiskurses selbst erneut angereichert durch spezifische lokale Ausdeutungen und Ansprüche. Dies ist nun zweifellos mehr als ein einseitiger Prozess der Translation. Treffender wäre das Bild einer Übersetzungsspirale: "The direction of a translation process is not linear, but more like a spiral with ideas moving from global to national to local to national to global. ${ }^{\text {"51 }}$ Ein solches Modell einer spiralförmigen Wechselübersetzung im global-lokalen Feld der Menschenrechte ist von politikwissenschaftlicher Seite als ein idealtypisches, linear konstruktivistisches „,spiral-

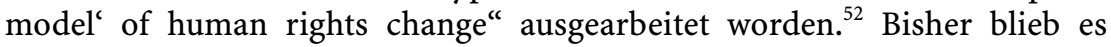

49 N. Rajaram u. Vaishali Zararia, Translating Women's Human Rights, S. 471. Zur Rolle von NGOs als „Vermittler und Übersetzer auf den nationalen Ebenen“ vgl. auch Shalini Randeria, Transnationalisierung des Rechts, in: WZB-Mitteilungen 101. 2003, S. 19-22.

50 Vgl. N. Rajaram u. Vaishali Zararia, Translating Women's Human Rights, S. 470.

51 Ebd., S. 481.

$52 \mathrm{Zu}$ diesem Spiralmodell vgl. Thomas Risse u. Kathryn Sikkink, The Socialization of International Human Rights Norms into Domestic Practices. Introduction, in: Thomas Risse u.a. (Hg.), The Power of Human Rights. International Norms and Domestic Change, Cambridge 1999, S. 1-38, hier S. 3. Auf diese Überlegungen von Thomas Risse 
allerdings überwiegend nur auf die lokale Implementierung von Menschenrechten in ihrem spiralförmigen Verlauf begrenzt.

Mit einer erweiterten und zugleich differenzierteren Vorstellung einer solchen Übersetzungsspirale hingegen ließe sich ein stärker handlungs- und kommunikationsorientierter Rücklauf beschreiben, der einen bis heute viel zu wenig beachteten Angelpunkt für eine translatorische Sicht des Menschenrechtsdiskurses markiert. Denn im Unterschied zum idealtypischen politikwissenschaftlichen Spiralmodell der Internationalisierung von Menschenrechten kommt damit nicht nur in den Blick, wie die Anwendung und Implementierung von Menschenrechten in jeweiligen staatlichen oder oppositionellen Handlungsfeldern über aufeinander folgende Phasen verläuft. Vielmehr wird erkennbar, dass der Horizont der globalen Menschenrechtsforderungen viel weiter ausgreift: diese erstrecken sich zwar zunächst bis auf die lokale Anwendungsebene, um dann jedoch von dort aus wiederum rückübersetzt zu werden auf eine transnationale Begründungs- und Erklärungsebene. Gerade über eine solche Übersetzungsspirale können lokal umkämpfte und erkämpfte Rechte wiederum neue Forderungen für den allgemeinen Menschenrechtskatalog bereitstellen.

Ein bekanntes Fallbeispiel sei hier angedeutet: das Einklagen von Menschenrechten beim Bau der gigantischen Staudammanlage entlang des heiligen Narmada Flusses in Indien. Der Bau dieses seit den 1980er Jahren vorangetriebenen, immer noch nicht abgeschlossenen, zugleich heftig umkämpften Staudammprojekts mit seinen dreißig großen und mehreren hundert kleineren Staudämmen soll der Energiegewinnung im Zuge industriellen Wachstums dienen. Er löste eine beispiellose gewaltsame Vertreibung beziehungsweise Zwangsumsiedlung der Bauern dieser Region aus, die mehr als eine halbe Million Menschen betraf. Durch Überflutung und Zerstörung der Wälder wurden die Existenzgrundlagen der dort lebenden Bauern vernichtet. ${ }^{53}$ Die Kanalisierung hatte zudem den Effekt, dass die elementare Ressource Wasser der lokalen Bevölkerung entzogen und einer übermächtigen Kontrollund Verteilungsgewalt unterstellt wurde. Schon deshalb richteten sich die Proteste der Bauern darauf, ihre Subsistenzbedürfnisse in Subsistenzrechte zu übersetzen und diese lokal einzufordern. ${ }^{54}$ In einem entscheidenden Schritt wurden sie dann ausdrücklich als Menschenrechte re-formuliert und in ökologische und ökonomische Rechte hinein übersetzt: in Land-, Forst- und Wasserrechte.

u.a. scheinen N. Rajaram und Vaishali Zararia mit ihrer Spiral-Formulierung anzuspielen, ohne dies jedoch offenzulegen.

53 Vgl. Christoph Dittrich, 20 Jahre Widerstand gegen das Narmada-Projekt in Indien. Südasien Informationen Nr. 11, Berlin 2006, http://archiv.ub.uni-heidelberg.de/savifadok/volltexte/2007/72/pdf/nr11_narmada.pdf.

54 Vgl. Wolfgang Sachs, Environment and Human Rights. Wuppertal Institute Papers Nr. 137, Wuppertal 2003. 
Gerade solche Rechtsansprüche blieben freilich nicht nur auf die lokale Erfahrungsebene begrenzt. Seit den 1990er Jahren wurde der Protest vielmehr transnational geführt, das heißt Vermittler/Übersetzer wie internationale NGOs gemeinsam mit Akteuren der indischen Öffentlichkeit wie der Schriftstellerin Arundhati Roy ${ }^{55}$ wirkten darauf hin, dass die lokalen Rechtsansprüche auf Umweltgerechtigkeit wiederum in den allgemeinen globalen Menschenrechtsdiskurs rückübersetzt werden konnten. Nicht ganz unbeeinflusst von diesem indischen Fall, der weltweite Diskussionen auslöste, ist sicher auch eine neuere Entwicklung im Feld der Subsistenzrechte. So wurde erst in jüngster Zeit, im Juli 2010, der Zugang zu sauberem Wasser von der UN zu einem kollektiven Menschenrecht erklärt und als 31. Artikel ergänzend in die UDHR aufgenommen. ${ }^{56}$

Das also, was gemeinhin unter Aneignung verstanden wird, ist ein höchst komplexer Vorgang. Er besteht nicht etwa nur aus einseitiger Lokalisierung, sondern zugleich aus dem Versuch, das Lokale selbst auf globale Bezugspunkte hin neu zu konzipieren. Übersetzung erweist sich dabei gewissermaßen als eine „(dis)locating practice“. ${ }^{57}$ Diese wirkt darauf hin, den Übersetzungsverlauf in eine Gegenrichtung umzulenken, von der lokalisierenden Umsetzung beziehungsweise Überarbeitung aus erneut in den allgemeinen Menschenrechtsdiskurs hinein. Dabei bleibt auch ein solch spiralförmiger Übersetzungsprozess - paradoxerweise - ein partielles Projekt. Schließlich mündet auch Übersetzung als „(dis)locating practice“ nicht darin - wie Sally Merry betont -, dass globale Ideen vollständig „eingebürgert“ werden: „The programs are appropriated and translated but not fully indigenized. To blend completely with the surrounding social world is to lose the radical possibilities of human rights “ ${ }^{58}$ Gerade durch eine nur partielle Lokalisierung und Aneignung menschenrechtsaktivistischer Programme behalten die Menschenrechtsforderungen auf lange Sicht ihr „radikales“ Vermögen, Veränderung herbeizuführen. Gerade indem sie handlungsmotivierende „Lücken“ offen lassen, bleiben sie als regulative Idee und unabgeschlossene Aufgabe interkultureller Aushandlung lebendig. Die konkrete Menschenrechtspolitik am Ort muss sich daran messen lassen.

55 Vgl. Arundhati Roy, The Greater Common Good, 1999, http://www.narmada.org/gcg/ gcg.html.

56 UNO-Resolution: Wasser wird zum Menschenrecht, in: SPIEGEL-Online, 28.7.2010, http://www.spiegel.de/politik/ausland/0,1518,708967,00.html.

57 Richard Edward u. Robin Usher, Glimpse Eight. (Dis) locating Practices - Mapping and Translating, in: dies., Globalisation and Pedagogy. Space, Place and Identity, London $2008^{2}$, S. $153-165$, hier S. 154: „We will suggest that mapping and translating are practices of (dis)location and also themselves impel a movement to (dis)locate practices; thus they are both a part of (dis) location and have the effect of (dis) locating. They are performative practices that (dis) order the world in various ways."

58 Merry, Human Rights, S. 178 u. S. 137. 


\section{Strategischer Universalismus}

Wohin also könnte eine Übersetzungsperspektive führen? Immer wieder wurde und wird die UN-Menschenrechtserklärung als das (westliche) Original für alle weiteren Diskussionen, Transformationen, Implementierungen und Erklärungen der Menschenrechte angenommen. Mit einem translatorischen Blick, der sich nicht zuletzt auch auf historische „pre-translations“ (wie etwa kolonialistische Konfigurationen) erstrecken sollte, lässt sich dieser Zusammenhang nun anders gewichten. Auch die vergangene und zukünftige Geschichte der globalen Menschenrechte könnte wohl am besten als Phänomen einer Übersetzung ohne Original beschrieben werden, nämlich als ein fortdauernder Translationsprozess.

In diesem Sinn hat die Ethnologin Anna Lowenhaupt Tsing die Geschichte der globalen Umweltschutzbewegung auf den Begriff gebracht: „In this sense of the term translation, there are no originals, but only a heterogeneous continuum of translations, a continual process of rewriting in which meaning - as well as claims of originality and purity - are made. ${ }^{\text {c59 }}$ In dem hier angedeuteten Fall geht es zwar darum, dass das herkömmliche liberale Muster der Umwelt- und Menschenrechte bei seiner lokal-regionalen Aneignung (besonders in Indien und Brasilien) in andere Bezüge hinein übersetzt wird: in Bezugsrahmen von sozialer Gerechtigkeit, Entwicklung und Kollektivansprüchen. Doch die emanzipatorische Möglichkeit dieses „rewriting“ liegt gerade darin, dass dabei - eben in Form der Umwelt- und Menschenrechte - immer noch ein universaler Referenzrahmen genutzt wird.

Ein solches Anschließen an universalistische Ideen ist auch in anderen Kontexten eine höchst wirkungsvolle kulturelle Praxis, die der Soziologe Martin Fuchs als „intentionale Übersetzung in ein drittes Idiom “60 bezeichnet hat. Aus seinen empirischen Feldforschungen zu den Dalit, den Unberührbaren in Indien, geht hervor, wie Übersetzungsstrategien für diese entrechtete Gruppe überlebensnotwendig sind: Um auf ihre eigenen Ausgrenzungen aufmerksam zu machen, übersetzen die Dalit ihre Marginalisierungserfahrungen ausdrücklich in einen universalistischen Bezugsrahmen globaler Idiome hinein. So versuchen sie, „eine neue religiös-sozialethische Sprache durchzusetzen [...], um ihre eigene Situation wie auch die Gesellschaft insgesamt $\mathrm{zu}$ transformieren ". ${ }^{61}$ Ein solches universelles Idiom bieten in diesem Fall der Buddhismus mit seinen Versprechen der allgemeinen sozialen

59 Tsing, Transitions as Translations, S. 253.

60 Martin Fuchs, Diskontinuierliche Prozesse. Die transformative Kraft der Übersetzung, in: Cristian Alvarado Leyton u. Philipp Erchinger (Hg.), Identität und Unterschied. Zur Theorie von Kultur, Differenz und Transdifferenz, Bielefeld 2010, S. 113-131, hier S. 124; vgl. ders., Reaching Out; Or, Nobody Exists in One Context Only. Society as Translation, in: Translation Studies 2. 2009, S. $21-40$.

61 Fuchs, Diskontinuierliche Prozesse, S. 118. 
Anerkennung, gleichzeitig aber auch die Diskurse von Zivilgesellschaft und Menschenrechten.

Auch an diesem indischen Fall ist auffällig, wie stark die sozialen Übersetzungsvorstöße einem "strategischen Universalismus“ folgen. ${ }^{62}$ Die Strategie ist, sich auf die universalen Menschenrechte zu beziehen, um damit an einen international anerkannten globalen Orientierungsrahmen anschließen zu können. Von ihm aus lassen sich dann die eigenen nationalen, lokalen und regionalen Interessen rechtfertigen und durchsetzen - um unübersehbar als Teil der globalen Zivilgesellschaft ernstgenommen zu werden, um Argumentationshilfen zu gewinnen und Netzwerke zu bilden:

Speaking - here, making claims and arguing cases - in terms of what are taken to be inherent individual and group rights provides an immediately recognizable translation of local crises into an increasingly widespread international legal language. ${ }^{63}$

Aus einer solchen Translationsbeziehung zwischen lokal/regional artikulierten Rechten und einem globalen rechtlichen Referenzrahmen - dies betont nicht nur der Ethnologe Donald Brenneis - könnte eine grundlegend neue Akzentsetzung in der Konzeptualisierung der Menschenrechte abgeleitet werden: Menschenrechtskonzepte verkörpern zwar wichtige Universalien im Sinn eines "flexible medium for translation", 64 ja eines handlungsaktivierenden Legitimationsrahmens, der eigene, lokale Menschenrechtsdebatten in Gang setzt. Auch dass man sich auf Menschenrechtsdokumente als Texte berufen kann, scheint unverzichtbar zu sein, um eigene Rechtsansprüche (ökologische, Land- und Eigentumsrechte) geltend machen zu können: „They use globalist texts to argue that global networks must pay attention to their own global wisdom“. ${ }^{65}$ Doch entscheidend ist, dass solche global-lokalen Zirkulationen innerhalb des Orientierungsrahmens der Menschenrechte konkrete Ansätze zu wechselseitigen, spiralförmigen Übersetzungsbeziehungen in einer pluralen Weltgesellschaft zugänglich machen. Auch wenn dabei die UN-Erklärung rhetorisch als Referenzpunkt - positiv oder negativ erhalten bleibt, scheint sich ein weitergreifender Horizont zu eröffnen, in dem sich ein „emerging original“ überhaupt erst herausbildet. „Emerging original“ - dies deutet auf eine transnationale Entfaltungsperspektive, auf einen Fluchtpunkt politischen Argumentierens und Handelns in einer globalen

62 Zum Konzept eines „strategischen Universalismus“ - in Anlehnung an Gayatri Spivaks Konzept eines „strategischen Essentialismus“ - vgl. Tsing, Transitions as Translations, S. $264-269$.

63 Donald Brenneis, Reflections. Toward New Conceptions of Rights, in: Charles Zerner (Hg.), Culture and the Question of Rights. Forests, Coasts, and Seas in Southeast Asia, Durham 2003, S. 219-234, hier S. 219.

64 Tsing, Transitions as Translations, S. 266.

65 Ebd., S. 269. 
Welt hin. In diesem Sinn hebt der Entwicklungs- und Umweltsoziologe Wolfgang Sachs hervor:

Human rights have changed their locus in the social imagination. Once the legal core of the post-war community of nations [eben in der UN-Declaration, D. B.-M.], they have now become the utopian horizon of international civil society. ${ }^{66}$

\section{Menschenrechte im Licht der Literatur}

Eine solche Verknüpfung von Menschenrechten mit „social imagination“ legt nahe, die Frage der Menschenrechte als Übersetzungsproblem auch auf utopische und emotionale Vermittlungsdimensionen zu richten. Gerade dafür könnten erstaunliche Anklänge an die Sphäre der Literatur fruchtbar gemacht werden. Zwar wird von geschichtswissenschaftlicher Seite aus das historische Erkenntnispotential von Literatur wie Literaturwissenschaft immer noch allzu leicht ignoriert. So wird auch kaum erkannt, dass Literatur eine wichtige Übersetzungsinstanz für Menschenrechte verkörpert. Doch eine Ausnahme bildet hier die Historikerin Lynn Hunt. In ihrem jüngsten Buch „Inventing Human Rights" behauptet sie, es sei der klassische Bildungsroman beziehungsweise das Genre der „epistolary novel“ um die Mitte des 18. Jahrhunderts gewesen, welche die Menschenrechtserklärungen überhaupt erst auf den Weg und ins öffentliche Bewusstsein gebracht hätten. ${ }^{67}$ Denn Individualrechte seien zuallererst von diesem literarischen Feld aus in allgemeine Menschenrechte übersetzbar gemacht worden, und zwar über die emotionale Brücke der Empathie. Dadurch würde im Vorgang des Lesens eine Identifikation mit Anderen als Gleichartigen ermöglicht. ${ }^{68}$ Solche Identifikationsangebote durch „compassion“ und „empathy“ finden sich - so Hunt - exemplarisch in Samuel Richardsons Romanen „Pamela“ (1740) und „Clarissa“ (1747). Heutzutage jedoch erscheint eine solche literarische Ausbildung von „moral sentiments“, die unter humanistischem Vorzeichen Menschenrechtsnormen übersetzt und sie durch bloße Lektüre verbreitet, in einem kritischeren Licht. Zunehmend werden die Schriftsteller selbst zu entschiedenen Menschenrechtsaktivisten. Sie demonstrieren die Arbeit der Menschenrechte „as a matter of storytelling ${ }^{\text {(669 }}$ und Literatur als „social work“, wie es Joseph Slaughter in seinem grundlegenden Buch zu den Allianzen zwischen internationalen Menschenrechten und „Weltromanen“ formuliert. ${ }^{70}$

66 Sachs, Environment and Human Rights.

67 Hunt, Inventing Human Rights, S. 40.

68 Ebd., S. 58.

69 Vgl. James Dawes, Human Rights in Literary Studies, in: Human Rights Quarterly 3. 2009, S. 394-409, hier S. 394.

70 Joseph R. Slaughter, Human Rights, Inc. The World Novel, Narrative Form, and International Law, New York 2007. 
Wie Lynn Hunt behauptet auch Slaughter, dass die programmatische Entwicklungsperspektive individueller Rechtsansprüche im Menschenrechtsdiskurs seit dem 18. Jahrhundert auf die humanistische Idee vom Bildungsprozess des Individuums zurückgehe. Sie wirke - ähnlich wie Literatur - als „enabling fiction“.71 So ist es sicher kein Zufall, aber doch erstaunlich, dass sich einzelne Delegierte bei der Abfassung von Artikel 29 der UN-Erklärung von 1948 in diesem Sinn - allerdings durchaus kontrovers - auf ein literarisches Paradebeispiel einer Persönlichkeitsentfaltung berufen haben: auf Daniel Defoes Roman „Robinson Crusoe“..$^{72}$ „Enabling“ meint gegenwärtig aber durchaus mehr. Mitzudenken ist der Impuls zu politischem Aktivismus durch „life narratives“, „testimonios“ und andere Formen von Selbstzeugnissen, ${ }^{73}$ wie ihn bekannte Schriftsteller und Schriftstellerinnen als Mittlerfiguren an den Tag legen: Arundhati Roy mit ihrem buchstäblichen Anschreiben gegen den Bau des schon erwähnten Narmada Staudamms ${ }^{74}$ ebenso wie die Friedensnobelpreisträgerin und Menschenrechtsaktivistin Rigoberta Menchú mit ihrem Eintreten für die Rechte der Quiché-Mayas in Guatemala, die sie in einem testimonio-Selbstzeugnis verarbeitet hat. ${ }^{75}$ Skandalös und bewegend ist der Fall des nigerianischen Schriftstellers und Menschenrechtsaktivisten Ken Saro-Wiwa, der über viele Jahre hinweg für die Rechte der Ogoni in Nigeria eingetreten ist - einer ethnischen Minderheit, deren Land die Mineralölfirma Shell jahrzehntelang ausbeutete und verseuchte, während sie unterdrückt wurde und verarmte. In diesem Kampf um die Rechte der Einheimischen wurde Ken Saro-Wiwa hingerichtet - trotz aller Berufung auf Menschenrechte und trotz der Verfassung einer eigenen Menschenrechtserklärung, der Ogoni Bill of Rights von $1990 .^{76}$

Obwohl an solchen Fällen die Grenzen eines Übersetzungsvermögens erreicht werden, das auf einem Aushandeln von Differenzen besteht, bleibt die politisch-utopische Zielvorstellung einer transnationalen Zivilgesellschaft insgesamt doch weiterhin auf das translatorische Projekt angewiesen. Erst eine kritische Übersetzungsperspektive macht es fragwürdig, wieweit Literatur und Menschenrechtsprogramme schließlich doch aus einem humanistisch aufgeladenen und kulturspezifisch verengten Projekt ausscheren müssten, um

71 Joseph R. Slaughter, Enabling Fictions and Novel Subjects. The Bildungsroman and International Human Rights Law, in: Publications of the Modern Language Association of America 121. 2006, S. $1405-1423$, hier S. 1406.

72 Ebd., S. 1405 f.

73 Kay Schaffer u. Sidonie Smith, Conjunctions. Life Narratives in the Field of Human Rights, in: dies. , Human Rights and Narrated Lives. The Ethics of Recognition, New York 2004, S. $13-34$.

74 Vgl. Arundhati Roy, Das Ende der Illusion. Politische Einmischungen, München 1999.

75 Rigoberta Menchú, I, Rigoberta Menchú. An Indian Woman in Guatemala [1984], London 2010.

76 Unter: http://www.waado.org/nigerdelta/RightsDeclaration/Ogoni.html. 
die Diskussion der Menschenrechte an eine globalisierte Weltgesellschaft anschlussfähig zu machen. Da diese eher von Differenzen als von globaler Empathie geprägt ist, wäre es vielleicht weiterführend, auch hier statt (idealistischer) „conjunctions“77 eher den Brechungen oder gar Abbrüchen von Übersetzungsprozessen auf die Spur zu kommen. Erstaunlicherweise gibt es dazu schon eine frühe Andeutung der Literaturwissenschaftlerin Barbara Harlow. Sie hielt die UN-Menschenrechtserklärung von 1948 zunächst ebenfalls für eine Übersetzung der Anliegen des klassischen Bildungsromans: „Its thirty articles translated the standard literary paradigm of individual versus society [...] The Declaration, that is, can be read as recharting the trajectory and peripeties of the classic bildungsroman ". ${ }^{78}$ Doch solange sich der Blickwinkel allzusehr auf das Individuum in seiner Selbstentfaltung verengt - so Harlows kritische Pointe -, würden andere wichtige Aktivitätsmuster für die Implementierung der Menschenrechte, wie etwa Intervention oder Widerstand, weitgehend ausgeblendet. Ein anderes Bild könnte sich ergeben, wenn man sich nicht nur entlang von „moral sentiments“ auf Literatur bezieht, sondern erst einmal am gemeinsamen Nenner von „narrative constructions“"79 ansetzt. Doch auch darüber hinaus verlangt eine Übersetzungsperspektive der Menschenrechte, nicht mehr in erster Linie nur Narrative und Texte als Übersetzungsmedien für Menschenrechte anzuerkennen und zu nutzen, sondern stärker als bisher auch neue Medien, digitale Internetforen und globale Technologien.

In der globalisierten Gegenwart haben Menschenrechte, wie sich zeigt, jedenfalls längst ihre traditionellen Rahmungen gesprengt: „human rights have spilled over their original container" ${ }^{80}$ Gerade dadurch eröffnen sich herausfordernde Horizonte, in denen die Übersetzungsfunktion der Menschenrechte noch produktiver werden kann: indem sie auf weitere, ganz anders gelagerte Diskurse übergreift.

77 Ebd.

78 Barbara Harlow, Barred. Women, Writing, and Political Detention, Hanover 1992, S. $252 \mathrm{f}$.

79 Zur Frage, wie „human rights function as narrative constructions“ und wie sie mit „story-telling“ als Ermöglichung von „self-representation“ der Subjekte verknüpfbar sind, vgl. Joseph Slaughter, Narration and International Human Rights Law, in: Comparative Literature and Culture 9. 2007, http://docs.lib.purdue.edu/cgi/viewcontent.cgi ?article $=1031 \&$ context $=$ clcweb\&sei-redir $=1 \#$ search $=\% 22 \mathrm{clc}+$ web + jospeh+slaughter+narration $\% 22$.

80 Sophia A. McClennen u. Joseph R. Slaughter, Introducing Human Rights and Literary Forms; Or, The Vehicles and Vocabularies of Human Rights, in: Comparative Literature Studies 46. 2009, S. 1-19, hier S. 13. 


\section{Translatorische Überschüsse des Menschenrechtsdiskurses}

Schon Eleanor Roosevelt, die Präsidentin der UN-Menschenrechtskommission, hat die Übersetzungsnotwendigkeit in der Menschenrechtsfrage ins Feld geführt. So schrieb sie am 22. Dezember 1948 in ihrer täglichen Zeitungskolumne „My Day“ mit Bezug auf die UDHR: „One should never belittle the value of words, however, for they have a way of getting translated into facts, and therein lies the hope for our universal declaration ". ${ }^{81}$ In der Zwischenzeit ist die Lage komplexer geworden. Die Übersetzungsdynamik der Menschenrechte ergibt sich nicht mehr gleichsam selbstläufig. Sie erschöpft sich kaum mehr in ihrer faktischen Verwirklichung. Auch ein bipolares Übersetzungskorsett zwischen westlichem Original und nicht-westlicher Kopie passt längst nicht mehr. Es weicht gegenwärtig einem Szenario, das Übersetzungen in vielerlei Richtungen erforderlich macht: in diskursive Praktiken und aktivistische Vorgänge hinein, in Konsensbildung und Partizipationschancen einer entstehenden Weltgesellschaft. Keinesfalls kann es mehr genügen, die universalen Menschenrechte als solche zu übersetzen. Statt auf Übersetzung von Menschenrechten könnte der Blick vielmehr auf Übersetzung durch Menschenrechte gelenkt werden, um Menschenrechte in ihren kontroversen Diskurslagen noch weitergehend als Ermöglichung eines universellen Referenzhorizonts zu nutzen. In einem ersten Schritt hierzu ist zu versuchen, die Frage der Menschenrechte selbst als einen Übersetzungskorridor auszugestalten - hin zu gänzlich anderen Konzepten, hin zu Rechten und Pflichten aus fernerliegenden Diskussionskontexten.

Für diesen Zusammenhang könnte es erneut hilfreich sein, mit Dipesh Chakrabartys wichtiger Unterscheidung zu arbeiten und Menschenrechtsübersetzungen als Fälle einer kritisch-strategischen „cross-categorical translation“ (eben nicht nur einer „cross-cultural translation“) aufzufassen. Denn sobald der Menschenrechtsdiskurs mit anderen Diskursen und ihren Kategorien Allianzen eingeht, kann er auch deren Konkretisierungs- und Realisierbarkeitspotential nutzen oder gar übernehmen: Die abstrakten Menschenrechtsforderungen werden dadurch an historische und sozial jeweils sehr unterschiedlich ausgestaltete, auf jeden Fall aber lokalisierte Konzepte anschließbar und somit handlungsnäher. Neben der schon erwähnten Wechselübersetzung mit dem literarischen Diskurs finden sich gegenwärtig weitere, höchst aufschlussreiche Kategorienallianzen beziehungsweise partielle Übertragungen von einer Sphäre in eine andere. Richtungweisend erscheint hier der Versuch, Fragen der Menschenrechte in konkrete Ausprägungen von Gedächtnispolitik hinein zu übersetzen und sie dadurch mit neuen, ausdrücklich auch historischen Handlungsbezügen anzureichern. Seien es Kolonialismuserinnerungen oder auch die jüngste „Globalisierung“ des Holocaust-Gedächt-

81 Eleanor Roosevelt, My Day, 22.12.1948, http://www.gwu.edu/ erpapers/myday/display doc.cfm?_y=1948\&_f=md001156. 
nisses - beides könnte der internationalen Menschenrechtsbewegung eine neue, historisch fundierte Triebkraft verleihen. ${ }^{82}$ Weitere translatorische Neuverknüpfungen bahnen sich an, wenn etwa Menschenrechtsfragen in den Entwicklungsdiskurs hinein übersetzt werden, was sich besonders in der Declaration of Development Rights niederschlägt. ${ }^{83}$ Umgekehrt wird auch die Übersetzung von Entwicklungsdebatten in Menschenrechtsdiskurse wiederum für die Menschenrechtspraxis fruchtbar. Denn auf diese Weise können Subsistenzrechte wie Nahrung, Wasser und Arbeit - wie schon beispielhaft gezeigt - als Menschenrechte re-formuliert werden. ${ }^{84}$ Jedenfalls lässt sich mit der Perspektive der Translation zugänglich machen, wie marginalisierte Bevölkerungsteile ihre Selbstermächtigung und Interessendurchsetzung vorantreiben können; etwa indem die Orientierung an Bedürfnissen in eine Orientierung an Rechten übersetzt wird: „The needs-centred approach is being replaced by a rights-centred approach ${ }^{\text {"85 }}$ - in ländlichen Gebieten zum Beispiel durch Reklamierung von Fischerei-, Land- und Forstrechten, in städtischen Gebieten beispielsweise durch Ansprüche auf Haus- und Wohnrechte oder auf Elektrizitätsversorgung.

Genau in diesem Sinn wird auch im jüngst diskutierten Feld von „spatial justice“ das Idiom der Menschenrechte neuerdings mit Raumkonzepten kombiniert, womit das Feld der Menschenrechte wiederum neu vermessen und in konkrete räumliche Rechte hinein übersetzt wird, zum Beispiel in städtische Strukturen, in Stadtrechte hinein. So erläutert der Raumtheoretiker Edward Soja, wie Menschenrechte in Stadträumen als „spatial justice“ reformuliert werden, ${ }^{86}$ wobei er Henri Lefebvres Idee von „le droit à la ville“ aufgreift: Räumlichen Gebilden wie Städten können ausdrücklich Rechte zugeschrieben werden. ${ }^{87}$ Die universellen Menschenrechte werden damit konkretisiert und politisiert; gerade durch ihre räumliche Übersetzung können sie politische Bewegungen auslösen, die dazu aufrufen, Haus- und

82 Vgl. Andreas Huyssen, Natural Rights, Cultural Rights, and the Politics of Memory. Hemispheric Institute of Performance and Politics, New York University 2009, http:// hemi.nyu.edu/hemi/en/e-misferica-62/huyssen?format=pdf.

83 Vgl. Andreas Eckert, Nachwort zu Hubertus Büschel u. Daniel Speich (Hg.), Entwicklungswelten. Globalgeschichte der Entwicklungszusammenarbeit, Frankfurt 2009, S. 311-319, hier S. 318: „Der Aufstieg von Entwicklungs- und Menschenrechtsdiskursen verläuft nahezu parallel, institutionell gibt es zahllose Überlappungen. Der Frage nach der gegenseitigen Prägung dieser Felder wurde bisher nur sehr unsystematisch nachgegangen."

84 Sachs, Environment and Human Rights, S. 26.

85 Ebd., S. 30.

86 Edward W. Soja, Seeking Spatial Justice, Minneapolis 2010, S. 32.

87 Allgemein zur räumlichen Neusicht von Rechten vgl. Franz von Benda-Beckmann u.a. (Hg.), Spatializing Law. An Anthropological Geography of Law in Society, Farnham 2009. 
Wohnrechte, Recht auf Elektrizitätsversorgung und andere lokal-räumliche Rechtsansprüche wahrzunehmen: „the notion of rights to the city, concretizing calls for universal human rights by embedding them in specifically urban spatial contexts and causalities, has been mobilizing multi-scalar political movements". ${ }^{88}$ Solche räumlichen Umsetzungen von Rechten reichen von Kämpfen um besseren Wohnraum und öffentliche Verkehrsmittel über Unruhen in den Banlieues französischer und anderer Großstädte bis hin zu den Protesten gegen den Bahnhofsbau von „Stuttgart 21“, ja sogar zum sich häufenden zivilgesellschaftlichen Widerstand gegen Fluglärm aufgrund von Einflugschneisen über Wohngebieten.

Ein weiteres, besonders aktuelles diskursübergreifendes Beispiel, das auch auf den jüngsten Klimagipfeln zur Geltung kam, ist die Übersetzung der Menschenrechte in die aktuellen Klimadebatten hinein: „Human-rights principles must be put at the heart of international climate-change policy making now, in order to stop this irreversible damage to humanity's future ". ${ }^{89}$ All diese hier nur angedeuteten Beispiele sind Übersetzungsfälle, die eine „cross-categorical translation“ fordern. Es sind Fälle von partiellen Übersetzungen, die sich gerade in ihrer Aufladung mit kulturellen und sozialen Erfahrungen in die andauernde Übersetzungskette beziehungsweise Übersetzungsspirale des Menschenrechtsdiskurses einklinken und die damit eine stärkere Wechselseitigkeit der Übersetzung zumindest stellenweise auch praktisch verwirklichen können. Solch unabgeschlossene Übersetzungen wirken zugleich darauf hin, Menschenrechtsdiskurse als ein transkulturelles Übersetzungsprojekt zu entfalten, das mögliche kulturenübergreifende Referenzrahmen aus den abweichenden kulturellen Positionen heraus überhaupt erst aushandelt. In dieser Richtung könnte sich die UN-Declaration weiterhin als ein „emerging original“ entfalten.

Worauf zielt also eine Übersetzungsperspektive in der Menschenrechtsfrage? Die lokale Umsetzung globaler Ideen und Programme ist sicher nur ein erster Schritt. Ziel ist eine grundlegende Veränderung der Art und Weise, wie überhaupt globales Wissen produziert und praktiziert wird. Etwas Entscheidendes nämlich fehlt bisher in der ganzen Frage der Übersetzbarkeit der Menschenrechte. $\mathrm{Zu}$ wenig wird bis heute darüber nachgedacht, welche Kommunikations- und Vermittlungsstrategien für ein globales Übersetzungsprojekt der Menschenrechte eigentlich gebraucht werden. Wir leben schließlich in einer Welt, in der die Instrumente und Standards der Verständigung selbst bereits deutlich voneinander abweichen. Das idealistische, westliche Konzept des „cross-cultural dialogue“, das in den Menschenrechts-Erklärun-

88 Edward W. Soja, Taking Space Personally, in: Barney Warf u. Santa Arias (Hg.), The Spatial Turn. Interdisciplinary Perspectives, New York 2009, S. 11-35.

89 Vgl. Oxfam Briefing Paper 117, Climate Wrongs and Human Rights. Putting People at the Heart of Climate-Change Policy, Oxford 2008, http://www.oxfam.org/sites/www.oxfam.org/files/bp117-climate-wrongs-and-human-rights-0809.pdf. 
gen immer noch durchscheint, kommt hier jedenfalls an seine Grenzen. Realistischer wäre ein kulturwissenschaftliches Konzept von „Übersetzung“, das ausdrücklich an Brüchen, Ungleichheiten und kulturellen Missverständnissen ansetzt und diese produktiv macht für ein Neuverständnis von Menschenrechten: Übersetzung nicht von, sondern durch Menschenrechte. Diese gelten dann weniger als statische Prinzipien, eher als eine „conceptual currency ${ }^{\text {(90 }}$ oder auch „language for deliberation ",, 11 mit der sich zunächst einmal überlagerte Kommunikationsschichten wieder zugänglich machen ließen. Doch dazu wäre nicht erst auf der Ebene des Dialogs anzusetzen, sondern bereits an Tiefenstrukturen wie pre-translations, an Referenzpunkten, an Übertragungsbedingungen in ihren historischen Vorprägungen und Verwerfungen: „translation-as-displacement “. ${ }^{92}$ In Bezug auf Menschenrechte heißt dies darüber hinaus: in einem rechtspluralistischen Umfeld disparate Problemfelder, Diskurse und Konzepte miteinander verknüpfbar zu machen. Gerade über solche Vernetzungsschienen wären die Menschenrechte auf verschiedensten Gebieten als unverzichtbare strategische Instrumente $\mathrm{zu}$ erkennen: zur Selbstauslegung ebenso wie zur Aktivierung sehr unterschiedlicher Referenzpunkte, zur lokalen Wissensproduktion, aber auch zur Handlungsermächtigung.

Dr. Doris Bachmann-Medick, International Graduate Centre for the Study of Culture (GCSC), Justus-Liebig-Universität Gießen, Alter Steinbacher Weg 38, D-35394 Gießen

E-Mail: doris.bachmann-medick@gcsc.uni-giessen.de

90 Kwame Anthony Appiah, The Ethics of Identity, Princeton 2005, S. 271.

91 Ebd., S. 264: „I prefer to see human rights as a language for deliberation, or argument, or some other form of conversation."

92 Chakrabarty, Place and Displaced Categories. 